\title{
exploitations à ciel ouvert
}

\author{
Rapport général de \\ L. Primel \\ L.C.P.C., Paris
}

Ce rapport ne vise en aucun cas à exposer tout ce qui a été fait en France dans le domaine des études géotechniques de carrières : les méthodologies d'études aux différentes échelles, depuis les inventaires jusqu'aux études détaillées de gisement, en passant par les essais géotechniques de laboratoire et de chantier qui permettent de définir l'utilisation des matériaux, ont fait l'objet de nombreux rapports, articles, normes (cf. Bibliographie); il s'agit plus concrètement d'essayer de montrer en quoi l'évolution des comportements dans un premier temps, puis de la législation plus récemment, ont eu des incidences importantes sinon sur nos méthodes elles-mêmes, en tous cas sur la façon de les mettre en cuvre.

La production de matériaux de carrières et plus particulièrement de granulats pose actuellement trois sortes de problèmes, que j'énoncerai dans un ordre qui n'implique aucune priorité :

a) Les premiers sont de caractère économique : compte-tenu du renchérissement des coûts de transport, il devient plus évident que jamais qu'il faut disposer de matériaux le plus près possible des lieux d'utilisation.

b) Les seconds - qui peuvent au premier abord apparaître en opposition avec les premiers - tiennent aux incidences multiples des exploitations sur l'environnement : ce sont les problèmes divers posés par les extractions dans les lits mineurs, ce sont les problèmes de pollution des eaux superficielles ou souterraines, ce sont les dégradations des paysages -, ce sont encore les nuisances diverses et classiques, comme le bruit, les poussières, etc.

c) Les troisièmes, complémentaires des précédents, et particulièrement aigus dans les vallées, sont les problèmes de concurrence pour l'occupation des sols : agriculture, urbanisme, exploitations forestières, etc.

Pour tenter de résoudre ces problèmes, se fait jour une tendance de plus en plus affirmée à pratiquer une politique prévisionnelle et une meilleure programmation des exploitations dans l'espace. Ces soucis divers se sont traduits par des lois qui modifient le Code Minier, et qui conduisent à une évolution importante dans la conception des études.

En quoi ces prises en compte nouvelles des problèmes ont-elles modifié les études géotechniques? Quelles ont été également les implications de ces modifications sur la recherche? Je n'en ferai pas une analyse détaillée, mais je donnerai quelques exemples:

$1^{\circ}$ Problème des extractions dans les lits mineurs des cours d'eau.

$2^{\circ}$ Les exploitations dans les lits majeurs : mise en place des zones d'exploitation et de réaménagement coordonnés.

$3^{\circ}$ Recherche de matériaux de substitution.

$4^{\circ}$ Les études d'impact au niveau des gisements et des réaménagements de sites dégradés.

\section{Extractions dans les lits mineurs}

De nombreux cours d'eau français charrient vers leur embouchure des matériaux qui se déposent dans des emplacements où la vitesse du courant est plus faible, et ces dépôts ont été depuis très longtemps exploités pour en extraire essentiellement des sables, sans qu'aucune étude particulière ait généralement été réalisée; on avait tendance à considérer qu'en enlevant les apports, l'industrie extractive favorisait l'écoulement des eaux et par conséquent celle des crues. On s'est aperçu assez récemment que l'extraction est très généralement supérieure aux apports : on estime que I'on extrait annuellement 12 millions de tonnes du lit de la Loire, alors que les transports de matériaux par le fleuve se limiteraient à 600000 tonnes. II faut ajouter en outre que contrairement à l'opinion couramment admise, le débit solide de la quasi totalité des cours d'eau français provient essentiellement des matériaux 
sablo-graveleux hérités des dernières glaciations et non de l'érosion continue des roches dans les massifs où ils prennent leur source.

Les extractions anarchiques et continues provoquent donc un creusement du fond du lit, qui a pour conséquences :

- l'abaissement de la ligne d'eau et par suite de la nappe phréatique, avec des conséquences possibles sur les captages et sur les rendements agricoles;

- l'affouillement des ouvrages en rivière : ce phénomène est particulièrement important pour les fondations de piles de ponts, notamment dans le cas de nombreux ouvrages anciens:

- l'érosion des berges. Bien qu'elle soit généralement due à de nombreuses causes, il est certain que les extractions ont dans certains cas, accéléré et aggravé le phénomène;

- les modifications du lit peuvent enfin avoir sur la faune et la flore aquatique ou voisine de l'eau diverses conséquences (dégradation ou suppression de frayères - destruction de flore ou au contraire implantation de nouvelles espèces désirées ou non, etc.);

- dans certains cas (Loire par ex.) remontée du bouchon vaseux et du front salé dans l'estuaire, avec ses incidences sérieuses sur l'alimentation en eau douce.

Cette prise de conscience de la gravité des problèmes posés par des extractions dans les lits mineurs a conduit d'une part à certaines interdictions et d'autre part à des études dans les domaines hydraulique, hydrogéologique et hydrobiologique beaucoup plus complètes qu'auparavant et qui permettent de définir dans quelles conditions on peut poursuivre les extractions et quelles sont les mesures à prendre (problème des seuils par exemple) pour éviter les conséquences que nous venons d'énumérer. On se soucie dans le même temps de beaucoup mieux maîtriser, non seulement la topographie du fond du fleuve, mais aussi l'épaisseur et la répartition spatiale des bancs de sables et graviers; ce qui suppose la mise au point, d'ailleurs en cours actuellement, d'appareils de mesure géophysiques simples permettant de définir rapidement ces paramètres en continu.

2 Devant les problèmes posés par les extractions dans les lits mineurs, le premier réflexe a été de transférer les exploitations dans les alluvions du lit majeur ou des basses terrasses des rivières. Seulement, il s'agit là d'un transfert dans un domaine qui est au moins aussi sensible sur le plan de l'environnement: n'oublions pas que les premières levées de boucliers contre les carrières se sont appuyées sur les pollutions et nuisances diverses créées par ces exploitations et qu'en outre elles se heurtent à la redoutable concurrence pour l'occupation des sols de l'agriculture, de l'urbanisme, etc.

Les conséquences dans le domaine géotechnique sont très importantes: alors que jusqu'à une date récente, on pouvait se contenter de délimiter les zones les plus riches en matériaux, puis de les exploiter, il devient maintenant indispensable de les concevoir dans un ensemble beaucoup plus complet : que les études se fassent dans le cadre de zones d'exploitation et de réaménagement coordonnées (Z.E.R.C.) ou par le biais de plans d'aménagement ou d'urbanisme, il s'agit en fait de pratiquer une politique prévisionnelle d'extraction, d'approvisionnement et de réaménagement. Les zones qui seront retenues pour l'exploitation devront être conçues dans un ensemble qui aura pris en compte les besoins locaux et régionaux, l'ensemble de ce que l'on a coutume d'appeler les " contraintes", et aussi, et c'est ce qui est le plus nouveau, d'intégrer les exploitations dans un cycle d'utilisation du sol, c'est-à-dire de prévoir le devenir de l'ensemble de la zone après exploitation. Sur le plan géotechnique, tous ces impératifs signifient que le gisement doit être parfaitement connu, encore mieux que lorsque le seul problème était d'extraire des matériaux; les variations d'épaisseur de la découverte et du matériau exploitable influent non seulement sur le choix des zones d'extraction, mais aussi sur les modalités ultérieures du réaménagement; le contexte hydrogéologique de l'ensemble du site devra être très bien maîtrisé, pas seulement pour les raisons classiques et évidentes (zones inondables, modalités d'extraction), mais aussi pour prévoir l'incidence des extractions sur le niveau et la qualité de la nappe, ce qui conditionne directement les futurs aménagements. II est bien évident que la plupart des méthodes à utiliser sont bien connues, mais cette évolution dans les préoccupations conduit cependant à une réflexion sur certains points de méthodologie. L'étude hydrogéologique consiste en fait à situer la place de l'extraction dans l'écoulement de la nappe, les influences étant différentes selon:

- sa pénétration dans la nappe, selon que l'on atteint ou pas le substratum de l'aquifère;

- sa position par rapport à l'aquifère et son type d'écoulement. Si l'extraction est située en amont de la nappe, elle peut contribuer à augmenter ou diminuer son alimentation (la notion « amont de la nappe » n'est d'ailleurs pas facile à définir avec précision);

- ses relations avec le cours d'eau;

- sa forme et la dimension de l'excavation par rapport à l'écoulement;

- les caractéristiques de laquifère (perméabilité, transmissivité, anisotropie, emmagasinement);

- la position de l'excavation par rapport à d'autres captages ou d'autres extractions.

En fait, il s'agit des paramètres nécessaires à l'étude hydrogéologique classique à l'échelle d'une nappe (et pas seulement à l'échelle du gisement). Les études peuvent se faire la plupart du temps par modélisation :

- modèles analogiques pour les cas simples.

- modèles mathématiques pour les cas plus complexes et les aquifères hétérogènes.

Outre ces études hydrogéologiques classiques (mais malgré tout différentes de ce qui se faisait auparavant pour des études de gisements alluvionnaires), des recherches sont engagées pour résoudre un certain nombre de points, soit insuffisamment connus, soit controversés, dont les principaux sont :

- Le colmatage du fond et des berges, correspondant à :

- l'accumulation côté aval, des fines entraînées par l'écoulement de la nappe.

- les éboulements et mélanges avec la terre végétale sur les bords,

- la production de fines lors de l'extraction,

- la prolifération d'algues sur le fond et les berges.

Ces éléments sont en fait assez mal maîtrisés actuellement et il est difficile de juger de leur importance et de leur évolution dans le temps :

- Évolution à terme de l'eau d'une extraction : 
- l'extraction entraîne une modification du rôle de filtre de l'aquifère, souvent complexe (suppression des écrans de surface, mise en communication de nappes superposées, etc.);

- si l'on constate qu'en général la mise à nu de lá nappe contribue à améliorer à court terme sa qualité par auto-épuration, on sait que ce phénomène est limité dans le temps par suite d'une pollution hydrobiologique qui dépend de nombreux facteurs, pas tous encore bien maîtrisés.

Citons encore les problèmes posés par le rejet (ou le recyclage) des eaux de lavage des matériaux extraits, ou le cas particulier, mais relativement fréquent des excavations en bordure de cours d'eau qui peuvent entraîner une modification du champ d'inondation, une rupture du cordon en cas de crue, un colmatage du cours d'eau par rejet des eaux de lavage, une modification de la température.

- Signalons enfin que certaines méthodes qui avaient été mises au point pour de nombreux types d'études géologiques se sont avérées particulièrement bien adaptées aux problèmes des gisements alluvionnaires: il s'agit en particulier des photographies aériennes obliques, dont l'interprétation apporte beaucoup d'éléments aussi bien pour l'étude du gisement que pour celle des écoulements souterrains, sans parler des données archéologiques, agricoles, forestières, etc. (cf. communication MM. Aubert-Gros).

\section{Les matériaux de substitution}

Les oppositions de plus en plus importantes aux extractions dans les lits mineur et majeur des cours d'eau, ont conduit peu à peu à envisager l'utilisation de matériaux dits de substitution. Qu'entend-on par matériaux de substitution? Schématiquement, il s'agit des matériaux susceptibles de remplacer les granulats alluvionnaires traditionnels dans leurs utilisations actuelles, c'est-à-dire essentiellement la fabrication des bétons et secondairement celle des matériaux de viabilité. Ce souci a conduit à la réalisation de nombreuses études, dont quelques-unes ont débuté dans certaines régions depuis plus de dix ans (calcaires tendres de la région parisienne, sables fins du Nord, par exemple), plus récemment dans le cadre de la taxe parafiscale sur les granulats, un peu partout en France.

On peut citer à titre d'exemples, les sables fins de la région parisienne, les schistes des terrils houillers du Nord, les calcaires tendres du Bassin de Paris au sens large, les alluvions argileuses des terrasses de la région toulousaine, les arènes granitiques et bien entendu les sables et graviers de la plate-forme continentale. L'élargissement considérable du champ des études a conduit à se poser toute une série de problèmes technico-économiques, dont les implications dans le domaine des études géotechniques proprement dites sont très importantes. Un rapide survol de quelques-uns des principaux matériaux de substitution permettra de s'en faire une idée:

- Matériaux alluvionnaires des moyennes et hautes terrasses, matériaux glaciaires et fluvio-glaciaires.

Cet ensemble très hétérogène correspond très généralement, soit à des placages plus ou moins importants sur les flancs des vallées, soit à d'anciens écoulements en nappes ayant laissé des dépôts très dispersés, soit enfin à des formations très hétérogènes (glaciaires et fluvio-glaciaires), mais souvent importantes localement.
Tous ces matériaux posent des problèmes beaucoup plus complexes que les gisements alluvionnaires classiques :

- la prospection est rendue difficile par les méthodes traditionnelles, en raison des variations très rapides et apparemment désordonnées des épaisseurs et des caractéristiques géotechniques (granularité, propreté);

- l'exploitation des gisements pose des problèmes de débourbage, de lavage, de recyclage des eaux pour des raisons de respect de l'environnement, et donc aussi de stockage ou réutilisation des boues de décantation;

- I'utilisation de ces matériaux ne peut se faire qu'après des études sur les formulations possibles (en particulier en fonction des possibilités de "neutralisation " des minéraux argileux).

- Sables et graviers de la plate-forme continentale. Leur exploitation pose encore un certain nombre de problèmes, surtout d'ordre économique. Sur le plan géotechnique, il s'agit essentiellement :

- de chercher à optimiser les méthodes de prospection à l'échelle de l'exploitation opérationnelle (le coût des sondages en mer est très élevé);

- de résoudre deux problèmes particuliers :

- quelle teneur en sel peut-on admettre pour les différentes utilisations?

- quel est le pourcentage admissible de débris coquillers?

- Granulats issus de calcaires tendres (ou, et) hétérogènes.

Très répandus en France, et en particulier dans des régions oủ les granulats alluvionnaires traditionnels se raréfient (Bassin parisien au sens large, Charentes, Jura), ils constituent certainement une des solutions de remplacement les plus rapidement opérationnelles. Mais leur exploitation systématique suppose d'abord que les recherches en cours aboutissent à des conclusions favorables :

- quelles sont les limites d'utilisation des différents types de calcaires tendres : problèmes de dureté, de granularité, de sensibilité au gel. Les études en cours sont plus complexes qu'on pourrait le penser, dans la mesure où les caractéristiques des granulats ne sont qu'un des paramètres intervenant dans le comportement du matériau composite;

- si les problèmes d'élaboration doivent pouvoir être résolus (pourcentage pas trop important de fines. élimination des « boulettes" d'argiles ou de marnes), il n'en reste pas moins qu'il est indispensable pour ce type de matériaux de rechercher les gisements présentant le moins possible de "points faibles" (hétérogénéités verticales : lits marneux, argileux, ou poches de dissolution remplies d'argiles, ou encore variations latérales rapides de faciès, silicifications discontinues, etc.). Les recherches de gisements sont donc plus délicates que dans les massifs homogènes, et toute méthode nouvelle efficace (telle la magnétotellurique artificielle) est la bienvenue dans ces contextes difficiles;

- le but de ce rapport n'étant pas de faire un inventaire exhaustif de tous les problèmes, il suffira pour conclure ce chapitre consacré aux matériaux de substitution d'énumérer brièvement les autres matériaux sur lesquels des recherches sont en cours: utilisation optimale des "sables" non alluvionnaires, en particulier des sables fins, utilisation des sables de 
concassage des roches magmatiques et métamorphiques pour la fabrication des bétons, "plein emploi " des "découvertes " de gisements, et en particulier des arènes granitiques, meilleure utilisation possible pour les éboulis divers, pour les déchets de mines, de carrières, les sous-produits industriels, tels que laitiers, scories, redéveloppement éventuel des granulats artificiels, etc. En fait, les problèmes divers, et en particulier les contraintes d'environnement, qui ont conduit à sortir de la facilité qui consistait à * écrémer n les gisements alluvionnaires ont donc conduit à une véritable explosion des études sur les matériaux de remplacement, et en tout premier lieu dans le domaine géotechnique.

\section{Les études d'impact de carrière et les réaménagements de sites dégradés}

La taxe parafiscale sur les granulats, instituée en 1975 , a comme objectifs principaux, d'une part d'assurer la continuité des approvisionnements, d'autre part de réaliser des opérations expérimentales, exemplaires ou curatives de réaménagement dans les zones qui ont été dégradées par les exploitations de matériaux. Plus de 100 sites ont été réaménagés dans ce cadre depuis 5 ans. Par ailleurs, le nouveau régime des autorisations d'ouverture de carrière, qui s'est traduit par le décret de décembre 1979, rend obligatoire l'étude d'impact. Le souci, dans les deux cas est le même; il s'agit de réinsérer dans le premier cas les sites dégradés dans leur environnement et d'inclure dans le second cas les extractions dans ce qu'on pourrait appeler un cycle d'occupation des sols. Nous avons vu tout à l'heure qu'au niveau des études plus régionales, ce souci était déjà très apparent, ce sont les futures zones d'exploitation et de réaménagement coordonnés. En ce qui concerne les études d'impact, la communication présentée aux Journées par MM. Archimbaud et Primel a pour but essentiel de montrer que tous les problèmes que l'on doit prendre obligatoirement en compte dans les études d'impact ne peuvent pas être traités de façon sérieuse et efficace sans une très bonne connaissance géotechnique du gisement. Réciproquement, l'existence même de cette nouvelle obligation conduit sinon à bouleverser, du moins à infléchir et compléter nos études géotechniques. Pour illustrer cette tendance, il suffit de reprendre les principaux paramètres qui définissent le gisement (qu'il s'agisse de roches massives ou de matériaux alluvionnaires).

- La découverte : jusqu'à présent il faut bien dire que dans les études de gisement seule était prise en compte une estimation globale de l'épaisseur de la découverte, dans le cadre d'une gestion économique du gisement et en particulier des terrassements. Le problème du "devenir" de cette découverte était rarement envisagé sérieusement, alors que maintenant il s'agit d'une notion essentielle

- il faut veiller à implanter judicieusement les stocks de découverte, en tenant compte de leur insertion dans le paysage, mais aussi de leur utilisation ultérieure éventuelle, par exemple pour la constitution de merlons qui serviront de protection contre le bruit ou contre les poussières, voire même d'écran de camouflage pour une meilleure intégration de la carrière dans le site;

- le stockage de la découverte doit aussi être organisé en fonction de ce qui est prévu dans l'étude d'impact pour la remise en état du site au fur et à mesure et en fin d'exploitation. Ces prévisions peuvent aller du simple remodelage, jusqu'à la reconstitution de terres agricoles, ce qui signifie qu'il faut disposer d'une identification qualitative suffisante des matériaux de découverte.

- Le matériau exploitable.

Jusqu'à présent, les zones inexploitables d'un gisement, et elles existent toujours, bien qu'ignorées au départ, étaient laissées telles quelles, et elles donnaient à la carrière un aspect détestable. Leur mise en évidence lors de l'étude du gisement doit au contraire permettre de les utiliser intelligemment, soit pour y placer les installations, les stocks, soit comme moyen de lutte contre le bruit, les poussières, mais encore faut-il que la prospection du gisement soit suffisamment précise pour qu'elles soient connues en temps utile.

- De la même manière, il faut bien dire que jusqu'à présent on ne se souciait guère de la répartition des hétérogénéités et de l'état de fracturation du massif, si ce n'est à la rigueur pour faire en sorte que l'on soit dans les meilleures conditions économiques et techniques possibles pour la réalisation des abattages. Or. l'étude d'impact doit apporter des données chiffrées sur les problèmes de vibrations en particulier si l'exploitation prévue se trouve à proximité de sites sensibles (cf. thème 2 des Journées et Visite de la Carrière de la Noubleau). Enfin de l'état de fracturation du massif́ dépendra l'état des talus et les conséquences pour la sécurité, ainsi que pour l'aspect paysager en fin d'exploitation et enfin de cette fracturation découlera la possibilité ou non de réaliser des banquettes ou des gradins et aussi de prévoir l'implantation de tel ou tel type de végétation, ou encore de réutiliser la carrière comme lieu de stockage (cf. communication M. Montjoie).

- Les études actuelles de gisement sont beaucoup trop légères dans le domaine de l'hydrogéologie. Ce constat est vrai pour les carrières de roches massives, et encore plus bien sûr pour les carrières alluvionnaires.

- La connaissance du battement de la nappe, qui suppose une étude étalée dans le temps (ce qui n'est pratiquement jamais fait jusqu'à présent) est importante, pour l'extraction bien entendu, mais aussi pour une réalisation correcte des travaux de remise en état en fin d'exploitation ou, ce qui est plus souhaitable, à l'avancement. On connait de nombreux sites où la méconnaissance de ce paramètre a été catastrophique, lors de remises en culture par exemple.

- L'évaluation du débit est également indispensable dans de nombreux cas, lorsque le lavage est obligatoire évidemment, ou lorsqu'on souhaite extraire hors d'eau et qu'un pompage est nécessaire, mais aussi pour l'utilisation future du site si l'on compte par exemple l'aménager en plan d'eau (ce qui est très fréquent en lit majeur). II s'agit dans ce cas d'évaluer si les circulations d'eau seront suffisantes, compte tenu des problèmes de colmatage évoqués ci-dessus, pour éviter entre autre une eutrophisation des eaux retenues dans l'excavation.

En conclusion, on peut dire que la prise en compte de l'environnement dans le domaine des carrières a obligé les géotechniciens à " lever le nez " du sol, à regarder plus loin, aussi bien dans l'espace que dans le temps; cette "ouverture" se traduit par une utilisation différente de nos méthodes traditionnelles, et aussi par l'obligation de lancer de nouvelles recherches (pour résoudre des problèmes aussi variés que le colmatage des extractions alluvionnaires, ou encore l'utilisation 
de calcaires tendres pour faire des bétons ou encore l'utilisation pour la prospection des gisements de nouvelles méthodes géophysiques, comme la magnétotellurique artificielle, ou enfin l'étude de la floculation des fines argileuses pour mieux résoudre le problème des eaux de lavage, etc.). Loin de les gêner, cette nouvelle dimension doit au contraire permettre aux géotechniciens de s'exprimer de façon plus complète.

\section{Références bibliographiques}

[1] Annales des Mines : numéro spécial "Carrières". Mars-avril 1981. 224 pages.

[2] C. Archimbaud, L. Primel; Connaissance du gisement et étude d'impact. Revue $\mathrm{Fr}$. de Géot. n 14 bis. Mars 1981.
[3] J. Aubert, G. Gros; La photographie aérienne pour la reconnaissance de l'environnement. Revue $\mathrm{Fr}$. de Géot. $n^{\circ} 14$ bis. Mars 1981.

[4] Bulletin de Liaison des Laboratoires des Ponts et Chaussées. Numéro spécial « Granulats ». Juin 1977.

[5] Les études d'impact de carrières de roches massives. Note d'information technique du Laboratoire Central des Ponts et Chaussées (59 pages). Septembre 1980.

[6] Les études d'impact de carrières de matériaux alluvionnaires. Note d'information technique du Laboratoire Central des Ponts et Chaussées (à paraître février 1982).

[7] A. Montjoie; Stockage de chaleur dans les excavations à ciel ouvert ou souterraines. Revue Fr. de Géot. n 14 bis. Mars 1981.

[8] C. Schrœder; Exploitation rationnelle d'un terril. Revue Fr. de Géot. n 14 bis. Mars 1981. 
